\title{
I mpacto de las fusiones bancarias en los clientes externos. Caso: Fondo Común Banco Universal-Venezuela
}

\author{
Muñoz, José* \\ Requena, Karen** \\ * Economista. MSc en Ciencias Administrativas, mención Finanzas, investigador y \\ profesor de la Universidad de Oriente, Cumaná-Venezuela. E-mail: imunozr@hotmail.com \\ Telefax 293-4513665. \\ ** Licenciada en matemáticas. MSc. en Ciencias Administrativas, mención Finanzas, \\ investigadora y profesora de la Universidad de Oriente, Cumaná-Venezuela. E-mail: \\ mscmrek@yahoo.es. Telefax: 58-293-4331730.
}

\section{Resumen}

En los últimos años, las instituciones financieras venezolanas han pasado por varias etapas, tratando de adaptarse a los cambios de la economía nacional. Inicialmente, pasaron de ser empresas financieras regionales a nacionales y luego de especializadas a universales, sin embargo, estas estrategias no resultaron del todo, teniendo que buscar otras alternativas como las fusiones. La investigación se orientó a determinar el impacto que causan las fusiones bancarias en sus clientes externos, tomando como caso específico a la entidad financiera venezolana: Fondo Común Banco Universal, para lo cual se utilizó una metodología de carácter descriptivo y una combinación de estrategia documental y de campo. Se aplicó un muestreo no probabilístico de tipo intencional, seleccionando los elementos con base en criterios o juicios de los investigadores. De acuerdo a la opinión generalizada por los consultados, el nuevo banco es percibido por los clientes como una entidad menos ágil a la hora de prestar sus servicios.

Palabras clave: Instituciones financieras, bancos, fusiones bancarias, clientes, fondo común.

The I mpact of Bank Mergers on External Clients: The Fondo Comun Universal Bank Case in Venezuela

\begin{abstract}
Over last years, Venezuelan financial entities have passed through several stages, in their attempt to survive changes in the national economy. At first, they passed from being
\end{abstract}


regional financial entities to a national status, and then from specialized entities to universal entities, however these strategies did not work out as planned, so they had to look for other alternatives, such as mergers. This paper was oriented towards determining the impact caused by bank mergers on their external clients, taking the particular case the Fondo Común Universal Bank in Venezuela. The method used was descriptive with a combination of documentary analysis and field work. Non-probabilistic intentional sampling was applied, selecting elements based on the criteria and judgement of the researchers. According to the general opinions of those interviewed, the new bank was perceived as a less agile entity in relation to the services offered.

Key words: Financial institutions, banks, bank mergers, clients, common funds.

Recibido: 02-07-22 . Aceptado: 03-07-0

\section{I ntroducción}

En los albores del siglo XXI, la economía se encuentra en una etapa de apertura comercial, donde los países andan en la búsqueda de nuevas fronteras partiendo de la integración de sus economías, que les permita mejorar sus relaciones comerciales y fortalecer sus ventajas competitivas. Gracias a la globalización, estas fronteras se han abierto, ayudando así a que los capitales fluyan sin ningún problema y las fuerzas del mercado sean las que la rijan.

En la actualidad, las empresas están en una constante reestructuración, tratando de adaptarse a los cambios. Señala Chacón (2000:32-34) que las instituciones financieras de Venezuela no han escapado a ello y en los últimos tres años se han visto involucradas en un intenso proceso de fusiones, con el propósito de mantener su cuota de negocio y de ser competitivas en un mercado que cada día se encuentra más atomizado por la banca extranjera, la cual en apenas diez años de operación en el país, abarcan el cuarenta y dos por ciento $(42 \%)$ de los depósitos del total del Sistema Financiero.

Grasso (2000:21-23) coincide con Chacón (2000:32-34) en que a mediados de 1998 el país cae en una de las recesiones más largas de la historia, cuyas características principales fueron; el achicamiento del mercado vía gastos públicos, declive del poder adquisitivo, fuerte baja de la demanda interna, aumento del desempleo, incertidumbre política ante un escenario electoral, entre otros. Todos estos factores contribuyeron a que la banca nacional, que tradicionalmente ha operado con exagerados márgenes de ganancia, tomara el camino de las fusiones, el cual consideró como el más expedito para mantener sus dimensiones, clientes y cuotas de mercado. Sin embargo, no fue sino hasta 
el 18 de mayo de 2000 cuando comenzó a materializarse de forma intensa este proceso, donde la mayor fusión registrada en la historia bancaria venezolana fue la que se anunció para esa fecha entre el Banco Unión Banco Comercial y Caja Familia Entidad de Ahorro y Préstamo. Esta fue seguida por otras de importante dimensión, tomando en cuenta factores como: los activos involucrados, la cuota de mercado tanto a nivel de captaciones como de colocaciones entre otros.

Es importante señalar que si bien es cierto que la fusión es un tema que viene siendo recomendado por los especialistas en materia financiera como la salida inmediata que tiene la banca para no caer en otra crisis, pareciera que al llevarse a cabo este proceso, las instituciones se interesan más por saber: ¿cuál será el nuevo activo total de la institución resultante?, ¿con cuántas agencias se manejará el nuevo banco? y ¿cuál será el monto de la cartera de créditos?; olvidando el factor esencial para su éxito, "el cliente", ya que sin él, no tendría sentido esforzarse por la producción y desarrollo de nuevos productos y mucho menos el de participar en un proceso tan complejo como el de unir dos empresas para formar una sola, con lo cual no se estaría alcanzando el objetivo del negocio, que no es más que el de maximizar la rentabilidad de los accionistas.

De allí la importancia que la banca tenga presente las exigencias de la clientela, ya que ésta representa su activo más importante. Expone González (2000:13-17) que el cliente es ahora más crítico y posee una mayor cultura financiera y tecnológica, requiriendo de un sistema capaz de personalizar la relación bancaria, mejorar la atención y la posibilidad de ofrecer nuevos servicios y productos y estar al día con el elemento tecnología. Esto implica servicios de mayor calidad, mantener la satisfacción del cliente, tener una plataforma tecnológica actualizada, no pasar por desapercibido la rapidez de respuesta y el respeto por el cliente $y$, principalmente, que cada relación, que cada contacto con la clientela, sea efectiva, ya que la misma irá exigiendo cada vez más un mejor servicio.

Siendo el cliente un factor tan importante y valioso para los negocios, el objetivo principal de la investigación consistió en determinar el impacto que han sufrido los clientes externos por las fusiones, tomando como caso específico la institución financiera venezolana Fondo Común Banco Universal, debido a que la misma ha pasado durante los dos últimos años por una serie de etapas, convirtiéndose de una Entidad de Ahorro y Préstamo Regional a una Entidad de Ahorro y Préstamo Nacional, alcanzando la categońa de Banco Universal a través de su unión con el Banco República Banco Universal en el mes de abril del año 2001, llegando a ser actualmente lo que conocemos como Fondo Común Banco Universal (en lo sucesivo Fondo Común). 
A pesar de lo interesante del tema, algunas factores limitaron la profundización del trabajo, entre ellos: lo limitado de los recursos, y lo cerrado que resultan las instituciones financieras a la hora de proporcionar información sobre sus clientes. Se consideró pertinente analizar en primer lugar el significado e importancia de las fusiones y de los clientes a fin de captar la atención del lector, y los resultados los presentamos a partir del análisis bibliográfico realizado y del estudio de campo aplicado.

\section{Materiales y Métodos}

Se utilizó una estrategia de estudio combinado, documental y de campo. La población estuvo representada por la cartera de clientes de Fondo Común (naturales y jurídicos), la cual está constituida actualmente por 631.577 clientes SUDEBAN (2000: 1), aplicando un muestreo no probabilístico de tipo intencional, que de acuerdo a Arias (1997:53) consiste en la selección de los elementos con base en criterios o juicios del investigador.

Debido a que no existe información sobre la calidad de los servicios que presta la institución bajo estudio y en función a la definición anterior, los autores se acogieron a realizar entrevistas focalizadas a 100 clientes externos, de los cuales 65 fueron personas naturales y 35 fueron personas jurídicas, tomando los siguientes criterios: a) clientes naturales y jurídicos con antigüedad no menor de dos años en cuentas corrientes y de ahorro; b) clientes naturales y jurídicos con saldos promedios entre cien mil y tres millones de bolívares durante los últimos seis meses, en cuentas corrientes y de ahorro; c) clientes naturales y jurídicos con operaciones crediticias vigentes (sólo pagarés); y d) clientes naturales y jurídicos con servicios de nómina; con lo cual se pretendió abarcar la mayor diversidad posible de clientes externos que conforman cualquier institución financiera.

Dadas las características del estudio, las técnicas utilizadas en la investigación consistieron básicamente en el análisis bibliográfico y en la realización de las respectivas entrevistas focalizadas. A efectos de obtener mayor veracidad en la información, hubo la necesidad de elaborar un instrumento con doble escala. En primer lugar y para la determinación de la opinión del cliente externo se estableció una escala de tipo ordinal de cinco puntos, ordenados desde un valor igual a uno hasta un valor igual a cinco, para opiniones que van desde "Muy Deficiente", hasta "Excelente" y segundo, a fin de definir el grado de satisfacción, se creó una escala de tres puntos, paralela a la de opinión, en la cual se solicitó al cliente externo que señalara el grado de importancia que tenía para él cada factor analizado. La creación de una doble escala permitió formular un modelo de evaluación ad hoc, similar al Modelo de Actitudes hacia el Comportamiento de Fishbein. El 
modelo creado se representa por medio de la siguiente ecuación: Donde S, (satisfacción) es una medida general de la opinión, (O), emitida por un individuo sobre un determinado factor, ponderada por la importancia, (Y), que para ese mismo individuo tiene el factor bajo estudio (Levin, 1979: 115) $\underline{\mathbf{1}}$.

Los autores están conscientes que el modelo supone intervalidad en las escalas utilizadas, las cuales en realidad son de tipo ordinal. Esta violación de las propiedades matemáticas de las escalas es común en estudios sociales, en los cuales se requiere hacer evaluaciones a datos de tipo cualitativo.

\section{Resultados}

\subsection{Fusiones Bancarias}

En tiempos actuales, fusión es una palabra que ya está incorporada al vocabulario cotidiano, ya que día tras día aparecen anuncios sobre empresas que se unen o que se compran unas a otras. Según afirma J ordan (citado por Gamboa et al, 2001: 132-152) las empresas cooperan por una necesidad mutua y comparten riesgos, con la finalidad de alcanzar un objetivo común, relativo a desplazar a los competidores o a tener la dimensión necesaria para sobrevivir en un mercado mundial. Una definición sencilla de fusión la expresan Weston y Brigham (1994: 1067) los cuales indican que no es más que “la combinación de dos empresas para formar una sola compañía" y de acuerdo a los mismos autores (1994: 1071-1072), la clasificación de los tipos de fusiones viene dada por: a) según su alcance en los proceso y b) según el procedimiento para la adquisición.

En tal sentido y de acuerdo a los procesos que se han dado en el país, se definen las fusiones bancarias como la unión de varias sociedades financieras que bien pueden ser especializadas (Entidades de Ahorro y Préstamo, Bancos Comerciales, etc.) o instituciones de categoría universal y se pueden clasificar: a) según la forma legal, unión que viene dada por la absorción en beneficio de una como lo es el caso del Banco Mercantil e Interbank, o por la creación de una nueva sociedad que sustituya a otras existentes, situación presente en el surgimiento de Unibanca Banco Universal; b) desde el punto de vista operacional y; c) según su grado de integración.

Las fusiones bancarias no son un proceso nuevo a nivel mundial, ya que si se habla de fusiones en estos términos se puede citar la del Chase Manhattan Bank y el Chemical Bank, la cual creó el mayor banco de los Estados Unidos de Norteamérica y el cuarto más grande del mundo. Dicha operación no fue más que un paso en la ola de concentraciones bancarias que había comenzado a principios del año 1995. Desde entonces se realizaron 
cinco grandes fusiones, que en opinión de los analistas, respondió a una evolución lógica, ya que ninguno de los diez mil bancos estadounidenses se situaba entre los veinte primeros del mundo (Agencia New York, 1995: 23-25).

En el caso específico del país, la banca nacional ha recorrido un camino accidentado si se toma en cuenta que la misma pasó desde la nacionalización bancaria de principios de los años 80 hasta la paulatina presencia y predominio de la banca extranjera los últimos años, aunado a la crisis de los años 1994-1995 que golpeó fuertemente al sistema bancario con el cierre de diecinueve instituciones financieras (Faraco, 2001:46). De allí que según Chacón (2000:32-34): “de ciento veinticuatro instituciones, hoy solo quedan ochenta y siete, de las cuales cinco concentran el cincuenta y seis por ciento de los depósitos del sistema y catorce aglutinan el treinta y tres por ciento, es decir, diecinueve bancos poseen más del noventa por ciento de los depósitos del sistema bancario nacional, debido a que previamente en los años 1996-1998 la estructura de la economía venezolana, la importancia del sector petrolero para el país y la forma de inyección de liquidez vía gasto público, crearon las condiciones para que se produjera un sobredimensionamiento del sistema financiero, ya que ante las expectativas de los futuros ingresos petroleros promovida por la llamada apertura petrolera, los bancos buscaron mantener el mayor nivel de captaciones posibles sobre los agregados monetarios esperados, e incrementar su cuota de participación en el mercado nacional". Lo anterior se puede visualizar a través de los Gráficos 1 y 2 .

El escenario que presentó el país a partir del año 1998 fue totalmente adverso a lo esperado, ya que sus principales características fueron: constantes fugas de capitales que sale del sistema financiero; altos costos de transformación y baja productividad con la que opera la banca nacional; baja rentabilidad que limita la expansión de la base patrimonial; incertidumbre nacional por la grave caída de los precios del petróleo, lo cual limitó considerablemente la inversión pública; expectativa de los inversionistas ante la proximidad de las elecciones presidenciales y; fuerte caída de la demanda agregada interna como consecuencia de la disminución del poder adquisitivo del venezolano (Datanalisis, 2001:7).

Aparte de todos estos factores que contribuyeron de cierta forma a que la banca tomara como camino más rápido e idóneo las fusiones, el gobierno nacional dio las bases legales que terminaron de impulsar y materializar estos procesos, mediante la denominada “Ley de Estímulo para el Fortalecimiento de los Gastos de Transformación en el Sector Bancario", comúnmente conocida como la "Ley de Fusiones Bancarias" publicada en gaceta Oficial № 37.076 de fecha 1 de noviembre de 2000, donde se brindan importantes 
facilidades a las instituciones que desean fusionarse.

Otro aspecto importante durante y después de los procesos de fusiones es la cultura organizacional y la atención al cliente; y para analizar este punto Maestre (2001, E-10), señala que: "el 35\% de las fusiones fracasan en el proceso de integración posterior. Las fallas posteriores al proceso de integración de las compañías objeto de las fusiones tienen que ver con el proceso de disfuncionalidad de las culturas organizacionales entendiendo por ésta como el conjunto de principios, creencias y normas de conductas aceptadas que determinan cómo funcionan las cosas en la empresa. Estos no suelen estar escritos y de hecho a la mayoría de las personas les costaría explicar lo que son, pero el conocer las normas y compartir las creencias define el nosotros y el que no las sigue queda clasificado como ellos".

Gräfico 1

Grado de Concentración de los Activos Financieros en Venezuela

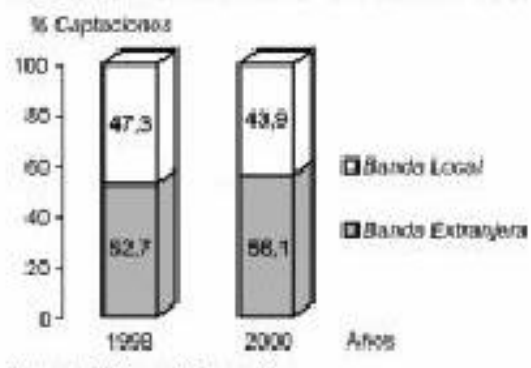

Fuente: Elaboración propia.

Gráfico 2

Número de Instituciones

Financieras en Venezuela
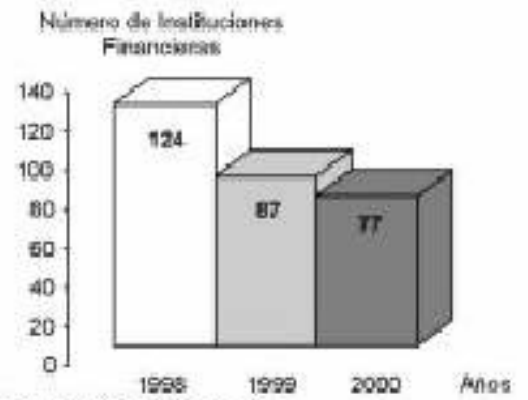

Fuente: Elaboración propia.

El mismo especialista expone que las empresas tienen tres opciones: a) imposición de una cultura sobre otra; b) permitir que las culturas coexistan, y; c) crear una nueva cultura a partir de las empresas que se fusionan. Esto lo que revela definitivamente, es que un proceso de fusión no se puede efectuar sin un arduo y delicado trabajo sobre la cultura organizacional y la atención al cliente. 
Lo anterior coincide con el estudio realizado por el especialista Dborkin (2001:E-11) quien indica que: "detrás de los movimientos de compra y venta se encuentra la cara menos visible, pero no por eso menos importante, del proceso: por una parte, las personas que, para bien o para mal, sienten que su vida laboral está a punto de sufrir un gran cambio y por la otra, las personas que de una u otra manera han estado recibiendo servicios de la institución y sienten desconfianza por la nueva organización que ha de crearse". Retomaremos esta tesis de Dborkin en nuestras conclusiones.

\subsection{Clientes-Fondo Común Banco Universal}

Abordar un tema donde se toca el factor cliente puede ser muy extenso y complejo, sin embargo, uno de los aspectos más importantes para el éxito de una empresa son precisamente sus clientes. Koontz y Weihrich (1998:54) sostienen que “ninguna empresa u organización podría existir sin ellos y para conseguirlos la compañía debe saber qué necesitan".

Es indudable que las expectativas y demandas de los sectores atendidos por las empresas se ven influidas por factores tanto no económicos (actitudes, deseos y expectativas de los individuos, derivados de los patrones culturales) como económicos, los cuales desempeñan un papel de gran importancia debido a que el cliente desea obtener las mayores ganancias por su dinero. Cabe destacar que las áreas como la administración y las relaciones humanas, son las que hacen énfasis en esta especialización.

Estudiar rasgos que diferencian los servicios de una institución de otra, supone definir el "estilo" particular de la organización que los realiza y la forma en que los clientes externos perciben los mismos. Para el caso específico de esta investigación se tomó una muestra de clientes externos de la institución financiera seleccionada (Fondo Común Banco Universal), entendiendo por éstos como todas aquellas personas naturales o jurídicas que mantienen algún tipo de instrumento bancario tanto activo como pasivo con la misma. De allí la importancia de precisar la misión y visión de la institución en estudio, considerando pertinente citar las declaraciones del presidente del banco, Sr. Fortunato Benacerraf, quien ha indicado que la misión es: "ser el banco venezolano preferido por la familia y la pequeña y mediana empresa, porque satisface con calidad y eficiencia sus necesidades financieras ofreciendo los productos y servicios que requieren", y la visión: "ser reconocido por su accesibilidad y trato amable, por su excelencia operativa y por su equipo humano entusiasta y cohesionado, que trabaja en un marco inquebrantable de valores éticos y morales, orientados a la solidez y rentabilidad de la institución".

De forma resumida, no es más que la maximización de la rentabilidad del negocio 
mediante el ofrecimiento de productos y servicios estándares que se adapten a las necesidades de su nicho de mercado a través de un personal calificado, lo cual puede ser considerada como la teoría básica bajo la cual operan las instituciones bancarias: maximizar las ganancias de los accionistas.

Ya definido los parámetros dentro de los cuales se ubica la institución seleccionada se cuantificó la calidad de los diferentes servicios que presta la misma luego de su fusión con el Banco República Banco Universal, por cuanto fue ello lo que permitió diagnosticar globalmente la calidad de la oferta de servicios de la institución analizada. Para el procesamiento de la información se establecieron los criterios de codificación pertinentes y se utilizó un programa de computador para la determinación de los resultados finales y para el análisis de la información se utilizaron modelos basados en estadísticas descriptivas, por lo que los resultados se evalúan tomando como base la frecuencia absoluta y relativa con que ocurren los valores de las variables bajo estudio.

En la Tabla 1 se detallan las ponderaciones dadas por los clientes a cada uno de los servicios evaluados. En ella es posible apreciar que los servicios que consideran de mayor importancia son: Disponibilidad y Efectividad de los Cajeros Automáticos, Asesoramiento al Cliente y Préstamos y Créditos. En contraposición a lo anterior, los resultados del estudio indican que el servicio de Home Banking (Internet) tiene una importancia relativamente menor, aún cuando estamos en la era de la tecnología.

El nivel de satisfacción de los servicios ofrecidos por Fondo Común Banco Universal se expone a continuación. 
Tabla 1

Importancia asignada por los clientes externos (NATURALES Y JURIDICOS) Fondo Común Banco Universal

\begin{tabular}{|c|c|c|c|c|}
\hline & $\begin{array}{c}\text { Muy } \\
\text { Importante }\end{array}$ & Importante & $\begin{array}{c}\text { Poco } \\
\text { Impertante }\end{array}$ & No contestó \\
\hline \multicolumn{5}{|l|}{ Ubicación Geogräfica } \\
\hline Ubicaciön & 25 & 19 & 43 & 13 \\
\hline Vias de Acceso & 16 & 22 & 51 & 11 \\
\hline \multicolumn{5}{|l|}{ Préstamos (Créditos) } \\
\hline Asescramiento & 55 & 32 & 3 & 10 \\
\hline Documentos Exigidos & 27 & 48 & 17 & 8 \\
\hline Rapidez de Respuesta & 75 & 12 & 0 & 13 \\
\hline \multicolumn{5}{|c|}{ Dispo. y Efec. de los Cajeros Autom. } \\
\hline Acceso & 68 & 23 & 0 & 9 \\
\hline Efectividad & 76 & 17 & 3 & 4 \\
\hline \multicolumn{5}{|l|}{ Asesoramiento al Cliente } \\
\hline Informaciön & 65 & 25 & 8 & 2 \\
\hline Cortesia & 80 & 15 & 0 & 5 \\
\hline Rapidez en el servicio & 79 & 18 & 0 & 3 \\
\hline Profesionalidad de los empleados & 55 & 39 & 6 & 0 \\
\hline \multicolumn{5}{|l|}{ Tarifa por los Servicios } \\
\hline Costo & 45 & 35 & 15 & 5 \\
\hline Informaciön & 32 & 42 & 22 & 4 \\
\hline \multicolumn{5}{|l|}{ Productos Ofrecidos } \\
\hline Información & 43 & 33 & 23 & 1 \\
\hline Innovaciones & 15 & 27 & 54 & 4 \\
\hline Tasas de Interés & 68 & 32 & 0 & 0 \\
\hline \multicolumn{5}{|l|}{ Servicio Telefónico al Cliente } \\
\hline Información & 45 & 35 & 15 & 5 \\
\hline Cortesia & 65 & 28 & 0 & 7 \\
\hline Prontitud & 72 & 18 & 4 & 6 \\
\hline \multicolumn{5}{|l|}{ Servicio Home Banking } \\
\hline Informacioin & 16 & 30 & 29 & 25 \\
\hline Acceso a la página web & 15 & 9 & 68 & 8 \\
\hline Costo & 18 & 11 & 45 & 26 \\
\hline
\end{tabular}

\subsubsection{Nivel de Satisfacción de los Clientes Externos-Jurídicos}

Préstamos (Créditos Bancarios): al evaluarse la forma como se concreta una operaci ón de crédito, desde su negociación hasta su liquidación, los encuestados reflejaron cierto descontento tanto por la gran cantidad de documentación exigida como por la tardanza en su liquidación (entre 40 y 45 dáas aproximadamente), desde que nace la operación hasta su abono en la cuenta del cliente, quien ha cumplido con todas las exigencias de la institución. Arrojaron unos porcentajes totales de $74 \%$ y $60 \%$ respectivamente en los renglones referidos a muy insatisfecho e insatisfecho (Gráfico 3 y 4). 


\section{Opinión sobre el SERvicio: Préstamos (Créditos Bancarios) \\ Fondo Común Banco Universal (Venezuela)}

Gráfico 3

Documentos Exigidos

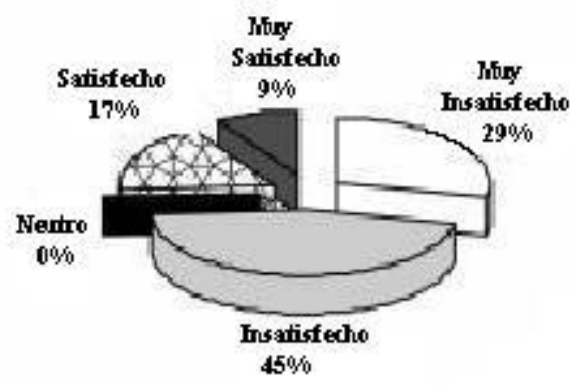

Gráfico 4

Rapidez de Respuesta

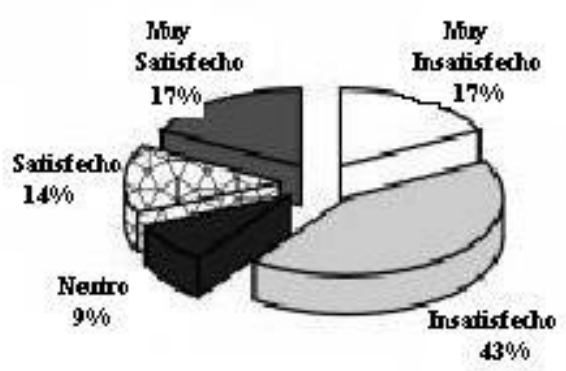

Fuente: Datos obtenidos a partir de la encuesta analizada

Asesoramiento al Cliente: de las variables consideradas en este servicio (Información, Cortesía, Rapidez en el Servicio y Profesionalidad de los Empleados) merece atención especial la evaluación dada a las variables de Cortesía y Rapidez en el Servicio, las cuales obtuvieron calificaciones desfavorables, $75 \%$ y $62 \%$ respectivamente. Cabe destacar que tanto el servicio que se le brinde a un cliente como la cantidad de instrumentos que éste mantenga con la institución, es lo que va a incidir en su permanencia o no en la misma (Gráficos: 5, 6, 7 y 8). 


\section{Opinión sobre el SERVICIO: Asesoramiento al cliente en el Fondo Común Banco Universal (Venezuela)}
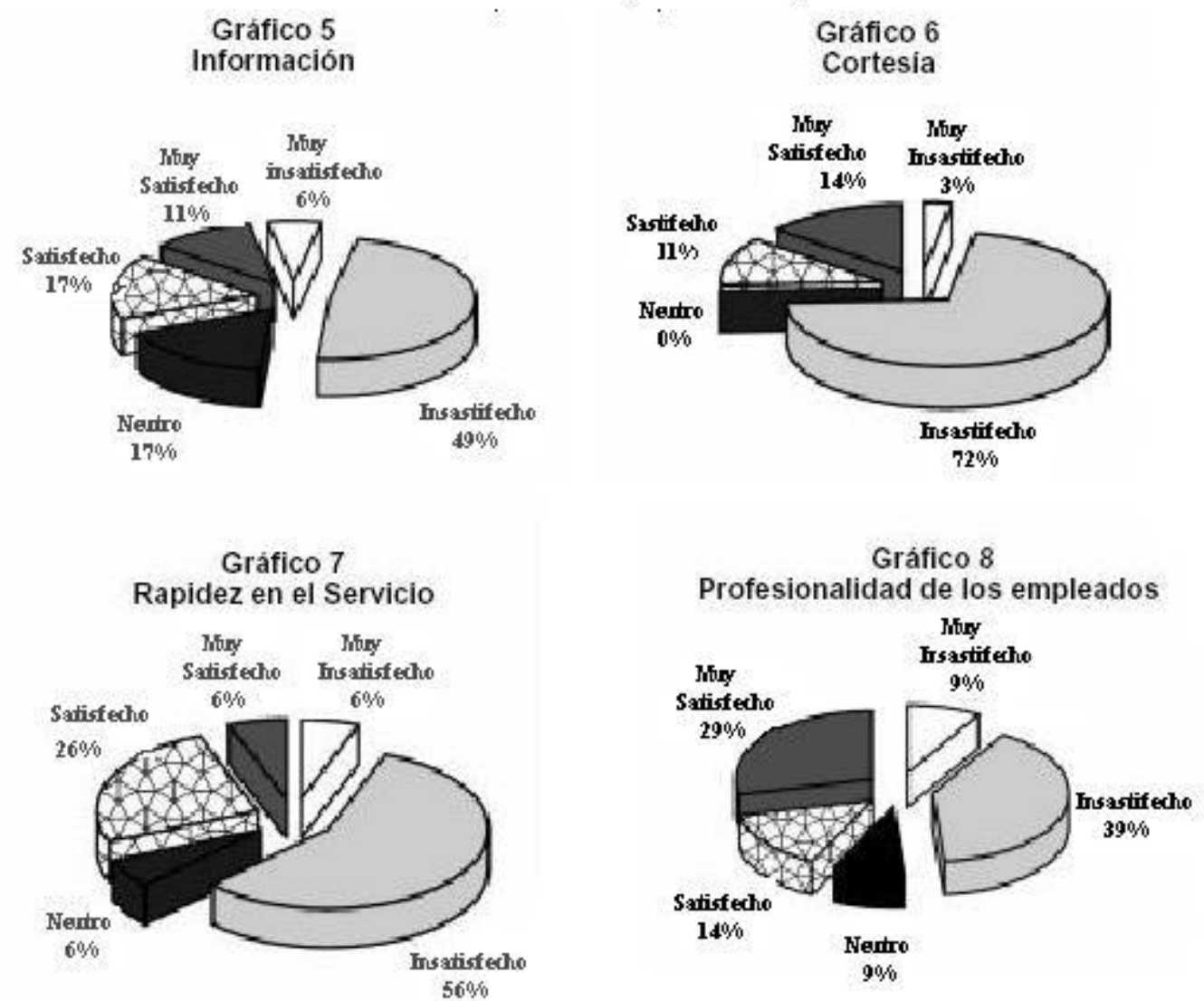

Fuente: Datos obtenidos a partir de la encuesta analizada

Disponibilidad y Efectividad de los Cajeros Automáticos: este servicio es evaluado con base en dos variables: facilidad de acceso, es decir, si se encuentran ubicados en determinados puntos a los cuales se les puede acceder por diferentes vías de la ciudad, y la efectividad o en que grado el cliente logra realizar satisfactoriamente la operación en el cajero automático. Los resultados de las mismas indican niveles de inconformidad del $63 \%$ y $60 \%$ respectivamente.

Tarifa por los Servicios: este servicio fue un factor evaluado desfavorablemente por la mayoría de los encuestados, ya que consideran muy elevada la comisión que cobra la institución financiera por los servicios conexos (cajeros automáticos, servicios de taquillas externas, elaboración de cheques de gerencia, transferencia, entre otros).

Productos Ofrecidos: las evaluaciones hechas por los clientes acerca de los productos ofrecidos tanto activos como pasivos, revelan niveles de satisfacción cercanos al $60 \%$ en cada una de las variables que lo componen (Información, Innovaciones y Tasas de Interés); en tanto que un porcentaje ligeramente menor manifestó insatisfacción y el 
resto se mostró neutral al respecto.

Servicio Telefónico al Cliente: la prestación de este servicio recibe evaluaciones favorables, ya que una gran parte de los clientes considera que son atendidos con cortesía y prontitud a la hora de realizar algún tipo de consulta de saldos o transferencia de fondos entre cuentas, por eso son catalogados como satisfactorios con porcentajes que oscilan alrededor del $75 \%$.

Servicio Home Banking (I nternet): las variables bajo estudio incluidas dentro de este servicio; Información, Acceso a la Página Web y Costos, reportan resultados que indican altos niveles de insatisfacción o desconocimiento por parte de los clientes. Las evaluaciones desfavorables o neutrales oscilan alrededor del $80 \%$; en tanto que las favorables en ningún caso superan el $23 \%$.

\subsubsection{Nivel de Satisfacción de los Clientes Externos-Naturales}

Préstamos (Créditos Bancarios): este servicio fue evaluado con base en dos variables: Documentos Exigidos y Rapidez de Respuesta. Los resultados indican niveles de insatisfacción elevados arrojando porcentajes de 54\% y 58\% respectivamente (Gráficos 9 y 10), ya que los clientes consideran que existen demasiados trámites desde el momento en que el cliente consigna toda la documentación exigida hasta la liquidación de la operación, sin embargo, es importante resaltar que la institución estudiada es una Entidad de Ahorro y Préstamo donde no se manejan las operaciones de pagarés.

\section{Opinión sobre el SERVICIO: Préstamos (Créditos Bancarios en el Fondo común Banco Universal (Nenezuela)}
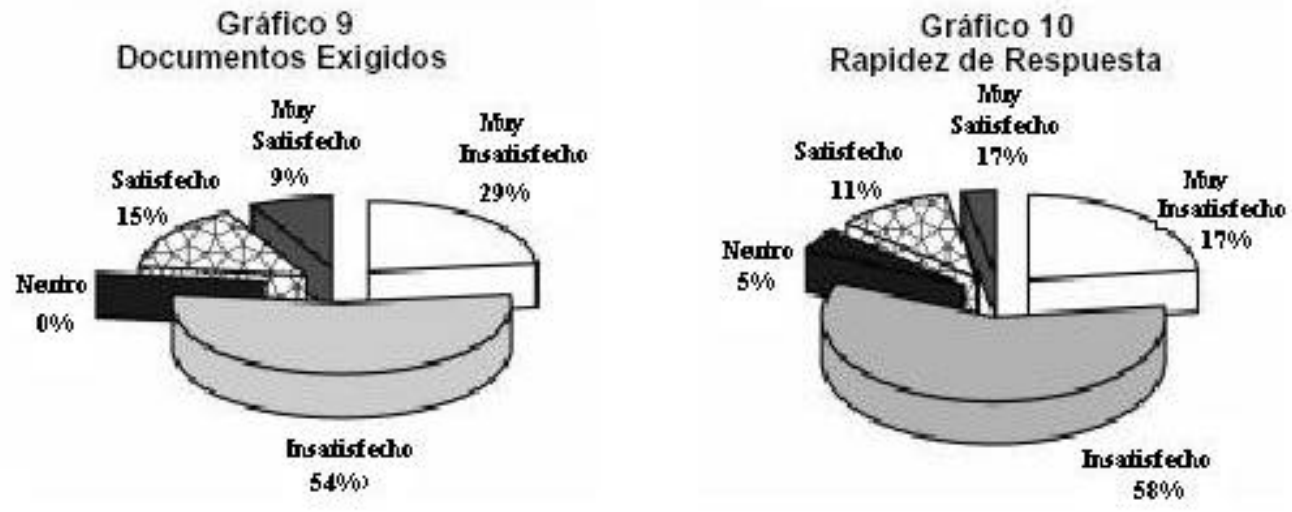

Fuente: Datos obtenidos a partir de la encuesta analizada

Disponibilidad y Efectividad de los Cajeros Automáticos: el servicio de Cajero Automático es, en general, considerado insatisfactorio o muy insatisfactorio. Consideran 
que los mismos están constantemente fuera de servicio ya sea por problemas técnicos o por falta de dinero, por lo que sería razonable analizar qué sucede al respecto.

Asesoramiento al Cliente: este factor es evaluado desfavorable por la mayoría de los encuestados, considerando que los empleados no se encuentran lo suficientemente informados con respecto a las bondades de los productos y servicios que la institución presta a la clientela. Por lo tanto, este punto merece especial atención, sobre todo cuando se ha pasado por un proceso de fusión, donde aparecen nuevos productos y servicios que tal vez difieran de aquellos que manejaban antes de la unión de las dos empresas (Gráfico $11,12,13$ у 14).

Tarifa por los Servicios: al igual que en el servicio anterior, los resultados de la evaluación del nivel de satisfacción de los clientes que utilizan los diversos productos que ofrece la institución, presentan altos niveles de insatisfacción, considerando que los mismos son costosos. De hecho, las autoridades de la institución consideran que los mismos se han incrementado en más de un $100 \%$ en el lapso de dos años.

\section{Opinión sobre el SERVICIO: Asesoramiento al Cliente en el Fondo Común Banco Universal (Venezuela)}

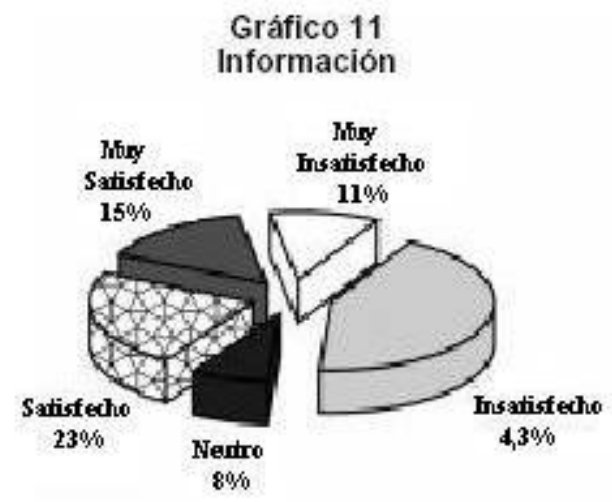

Gráfico 13

Rapidez en el Servicio

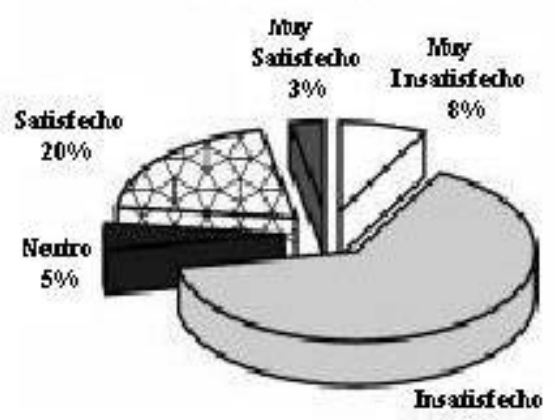

$64 \%$

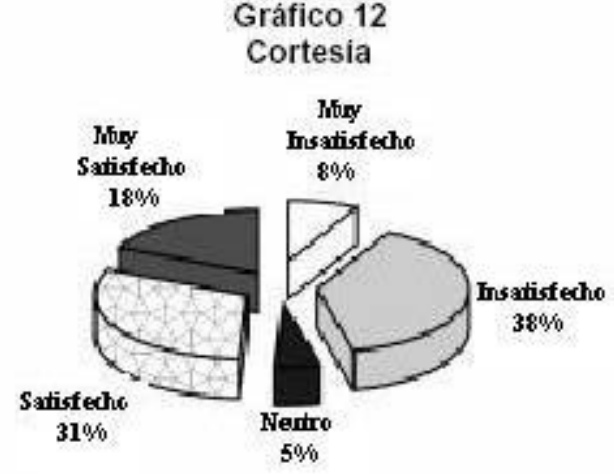

Gráfico 12

Cortesia

Gráfico 14

Profesionalidad de los Empleados

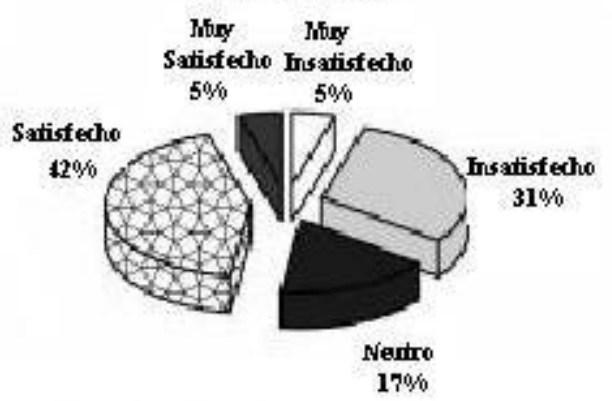

Fuente: Datos obtenidos a partir de la encuesta analizada 
Productos Ofrecidos: las variables evaluadas en este servicio, al igual que en los clientes externos jurídicos, obtuvieron altos niveles de satisfacción; no obstante las tasas de interés reciben una calificación más baja, en la cual el $54 \%$ de los clientes manifestaron insatisfacción.

Servicio Telefónico al Cliente: de las variables evaluadas en este servicio, más del $75 \%$ de los clientes calificaron como satisfactorio o muy satisfactorio: la Información, Cortesía y Prontitud, reflejando que el servicio funciona.

Servicio Home Banking (I nternet) : las variables bajo estudio incluidas dentro de este servicio; Información, Acceso a la Página Web y Costos, reportan resultados que indican altos niveles de insatisfacción, ya que muchos consideran que al abrir la página web, esta tarda demasiado, quizás por la cantidad de imágenes que posee. En todos los casos, las evaluaciones desfavorables o neutrales oscilan entre $65 \%$ y $80 \%$; en tanto que las favorables en ningún caso superan el $20 \%$.

\section{Conclusiones}

El objetivo principal de la investigación consistió en determinar el impacto que han sufrido los clientes externos de Fondo Común Banco Universal después de la fusión, los hallazgos obtenidos tienen importantes implicaciones. Si analizamos las valoraciones dadas por los clientes a las variables seleccionadas, encontramos que tanto los clientes jurídicos como los naturales otorgan puntajes muy similares a los mismos aspectos evaluados, es decir, un alto porcentaje de la muestra consideró que los factores Créditos (préstamos bancarios), Disponibilidad y Efectividad de los Cajeros Automáticos, Asesoramiento al Cliente, Tarifa por los Servicios y el Home Banking no se prestan de la forma más adecuada, pero contrario a esta situación, las valoraciones dadas a los servicios Telefónicos, Productos Ofrecidos, Acceso a los Cajeros Automáticos fueron bastante positivas.

Es evidente que la integración del cliente debe vincularse estrechamente con la visión de la organización. Uno de los aspectos que resulta más difícil de controlar es el grado de satisfacción del cliente, particularmente resulta complicado medir si el desempeño de los empleados contribuye o no para lograrlo. De allí, que si la institución tiene como meta principal convertirse en el banco de la familia venezolana a través del ofrecimiento de excelentes productos, buen trato al cliente y rapidez de respuesta; tanto la gerencia como los empleados en general deben prestar mayor atención a los servicios que ofrecen a fin de mantener su competitividad. La capacitación es una de las soluciones. Otra es observar los métodos de los competidores para servir a sus clientes y por último la satisfacción del 
cliente debe ser objeto de una vigilancia sistemática.

Una vez que se ha concretada la unión de las dos empresas, el reto de los que coordinan dicho proceso debe continuar hasta que la nueva empresa logre funcionar de forma adecuada y genere las utilidades que esperan sus accionistas mediante la adquisición de un mayor valor de mercado al que tenían las partes que se integraron, lo cual se conoce comúnmente como sinergia.

Retomando la tesis de Dborkin (2001), a la hora de realizarse una fusión, se debe tener en cuenta tanto los aspectos cualitativos como los cuantitativos, lo cual ha de traducirse en perjudicar lo menos posible al cliente, que de por sí ya ha sido impactado al enterarse de que el banco con el cual tal vez lleva una relación de años y hacia el cual hasta puede tener un alto sentido de pertenencia, está a punto de desaparecer o de realizar importantes cambios, sin dejar de hacer mención del personal interno quién también sienten que su vida está a punto de cambiar.

En definitiva de acuerdo a la opini ón generalizada por los consultados, a) El nuevo banco es percibido por los clientes como una entidad menos ágil a la hora de prestar sus servicios, frente al banco antes de fusionarse. b) El actual banco ofrece intereses más competitivos para los depositantes, más no así para los créditos, lo cual coincide con la tónica general del sector bancario y c) El Profesionalismo de los Empleados es bajo, lo que da a entender que los mismos no están dando lo mejor de sí a la nueva institución.

Como dijimos en la discusión, el factor esencial para el éxito de cualquier negocio es el cliente, ya que sin él no tendría sentido los esfuerzos de producción y desarrollo de productos y en fin, no se alcanzaría el objetivo del negocio que es el de maximizar la rentabilidad para los accionistas. No obstante, en muchas ocasiones el cliente no es considerado como aspecto importante en las fusiones y el mejor ejemplo de ello es que en los elementos que se analizan para la valoración del proceso de fusión, no suele tomarse en cuenta la pérdida de los mismos en la nueva empresa resultante.

A manera de reflexión, y de acuerdo a las conclusiones alcanzadas a través de esta investigación se pudo constatar que los clientes tienen una visión diferente de la que puede tener cualquiera que no trabaje en esa institución. Así pues, la estrategia de mercadeo de una entidad financiera deberá ir encaminada a aproximar la percepción de los atributos de la realidad, es decir, que no existan diferencias entre lo que piensa visión ofrecida por los no clientes - y lo que realmente es - visión ofrecida por los clientes. Es más fácil retener a un cliente que captar a uno nuevo. 
1. Las características del modelo son: La escala ordinal para solicitar la opini ón sobre los factores, fue definida como (1) muy deficiente, (2) deficiente, (3) regular, (4) bueno y (5) excelente, se modifico a: - 2, -1, 0, 1 y 2, para sustituir los valores que van desde 1 al 5 respectivamente. Al ponderar las opiniones reportadas por los encuestados, debido a la importancia dada al factor bajo estudio ( $1=$ Poca importancia, $2=$ Regular y 3 = mucha importancia); surgen las siguientes opciones: Alta satisfacción $=$ valores iguales a 6,4 ó 3; Satisfacción media = valores iguales a 2 ó 1; Neutro = valor igual a 0; Insatisfacción media = valores iguales a -2 ó - 1 e Insatisfacción alta = valores iguales a -6, - 4 ó -3 . Dado que los valores 2 y -2 pueden ocurrir en dos formas (2* 1 y $1 * 2)$ y ( $-2 * 1$ y $-1 * 2)$; y por cuanto el valor 0 puede ocurrir tres veces; es evidente que la escala contempla probabilidades iguales para cada evento ocurra en forma aleatoria; lo cual permite suponer que los resultados de la aplicación de la encuesta responden al valor real de la variable y no a factores al azar.

\section{Referencias Bibliográficas}

1. Agencias New York (1995), Fusión de Chase Manhattan y Chemical. La Jornada. Agosto 29. Nueva York.

2. Arias, Fidias (1997), EI Proyecto de Investigación-Guía para su elaboración. 2da Ed. Editorial Episteme, C.A. Venezuela.

3. Asamblea Nacional (2000), Gaceta Oficial No. 37.076. Ley de Fusiones Bancarias o Ley de Estímulo a la competitividad, el Fortalecimiento Patrimonial y Racionalización.

4. Fondo Común Banco Universal (2001), Boletín Informativo. Año 1, Número 1. Caracas.

5. Chacón, Gerardo (2000), Banca-Fusiones Bancarias a la Carta. Revista Dinero (27). Caracas.

6. Dataanalisis (2001), La Visión Integral-La Economía Venezolana en el 2001.

7. Dborkin (2001), Proceso de Fusiones, El Nacional. Agosto (15). Caracas.

8. Faraco, J. (2001), La Actividad Financiera. Un Proceso decadente en Venezuela. Revista Business (7). Caracas.

9. Gamboa, Teresa; Arellano Madelein y Nava Yuneska (2001), Estrategias Empresariales: Aproximación a una Tipología. Revista Venezolana de Ciencias Sociales, UNERMB. Vol. 5, No. 2. Maracaibo. 
10. Grasso, José (2000), Furor Bancario. Revista Dinero (22). Caracas.

11. Gonzalez, Enrique (2000), Fusiones en Venezuela. Revista Venezuela Analítica (12).

12. Koontz, Harold y Weihrich, Heinz (1998), Administración. Una Perspectiva Global. 11va Ed. Editorial Mc Graw Hill. México.

13. Levin, John (1079), Fundamentos de Estadística en la Investigación Social. Ediciones Harla. México.

14. Maestre, Raúl (2001), Fusiones y Cultura Organizacional. El Nacional Caracas.

15. SUDEBAN (2000), Indicadores para la evaluación del Sistema Financiero al 31 12.2000. http://www.sudeban.organization ve. Consulta: Febrero 15/2002. Internet.

16. Weston, Fred y Brigham, Eugene (1994), Fundamentos de Administración Financiera. 10aㅡ ED. Editorial McGraw Hill. México. 